\title{
Social Network Analysis of COVID-19 Public Discourse on Twitter: Implications for Risk Communication
}

\author{
Paola Pascual-Ferrá, PhD; Neil Alperstein, PhD; Daniel J. Barnett, MD, MPH
}

\section{ABSTRACT}

Objectives: The purpose of this study was to demonstrate the use of social network analysis to understand public discourse on Twitter around the novel coronavirus disease 2019 (COVID-19) pandemic. We examined different network properties that might affect the successful dissemination by and adoption of public health messages from public health officials and health agencies.

Methods: We focused on conversations on Twitter during 3 key communication events from late January to early June of 2020. We used Netlytic, a Web-based software that collects publicly available data from social media sites such as Twitter.

Results: We found that the network of conversations around COVID-19 is highly decentralized, fragmented, and loosely connected; these characteristics can hinder the successful dissemination of public health messages in a network. Competing conversations and misinformation can hamper risk communication efforts in a way that imperil public health.

Conclusions: Looking at basic metrics might create a misleading picture of the effectiveness of risk communication efforts on social media if not analyzed within the context of the larger network. Social network analysis of conversations on social media should be an integral part of how public health officials and agencies plan, monitor, and evaluate risk communication efforts.

Key Words: COVID-19, risk communication, social media, social network analysis, World Health Organization (WHO)

$\mathrm{M}$ ost primers on risk communication emphasize the need for public health officials and health agencies to communicate information in a timely, consistent, and clear manner as an effective strategy for disease prevention and preparedness. ${ }^{1,2}$ According to the World Health Organization (WHO), risk communication is essential to help people understand how to protect themselves, stop the spread of disease, and limit the social and economic impact of an outbreak. ${ }^{3-5}$ Risk communication disseminated by public health officials and agencies, however, is not received in a vacuum; rather, it becomes part of a larger ecosystem that includes information from multiple sources, such as friends, family, media, opinion leaders and influencers, among others. Social media, in particular, plays an important role in successfully communicating risk to the larger public. Twitter, in particular, is seen as the leading public communication platform for world leaders ${ }^{6}$ and an essential medium for disseminating public health information during outbreak situations and pandemics. ${ }^{7-14}$ A 2018 Pew Research Center study found that $71 \%$ of Twitter users in the United States get their news from Twitter. ${ }^{15}$

Studies looking at how pandemic-related information spreads on Twitter found that while users tend to favor reputable sources of information, many will share information lacking in sound scientific evidence. ${ }^{11,12}$ Conflicting messages from multiple sources of information can lead to increased confusion, higher levels of anxiety and additional negative impacts on mental health, not to mention misguided health behaviors, among the public. ${ }^{16-21}$ They may also increase the likelihood that individuals will act driven by fear rather than by medical guidance from health authorities, and increase skepticism in information sources. ${ }^{22-24}$ Furthermore, mismanagement of risk communication can decrease the credibility of the public health officials and agencies whom people trust with public health and safety. ${ }^{25}$

Monitoring public discourse on social media during a pandemic situation is critical to evaluate the effectiveness of risk communication efforts. ${ }^{14}$ Understanding what topics and messages are driving the conversation and who are mediating conversations is one way of evaluating their impact. ${ }^{26}$ Recent research examining discourse on Twitter around coronavirus disease 2019 (COVID-19) has focused on extracting themes from tweets mentioning "Coronavirus" and "COVID-19,"27 analyzing the most-liked tweets made by world leaders ${ }^{28}$ and looking at the spread of information and news frames 
of top shared sources. ${ }^{29}$ On Twitter, the most visible quantitative metrics to measure the impact of a tweet include the number of replies, retweets, likes, mentions, impressions, reach, and use of a specific \#hashtag. Qualitative monitoring includes doing sentiment analysis of tweets, comments, and replies. The latter, however, tend to focus on the actions of individual users and offer a partial view of the impact of communication in a network. A social network approach, on the other hand, can provide a bird's eye view of public discourse online. Social network analysis (SNA) uses graph theory to represent the structure, makeup, and interaction between members of a network. ${ }^{30,31}$ When applied to conversations on social media, SNA is useful for understanding the impact risk communication can have on a network.

Successful risk communication requires that public health officials and agencies lead the conversation and ensure that the public receives accurate, science-based information when it is most critical. Given the importance of coordinated communications during a pandemic situation, ${ }^{3-5}$ a consistent and unified message that is amplified or reiterated across a network may help increase public awareness about ways to slow the spread and reduce the impact of a virus. The alternative, the fragmentation or atomization of public discourse into clusters of smaller, potentially competing conversations, can dilute the message and reduce the effectiveness of risk communication efforts. Nahon-Serfaty defined this fragmentation of discourses in health communication as "a complex dynamic nourished by competitive and opposite views about diseases' causes and risk factors, preventive measures, and therapeutic solutions, in the context of globalized media and hyper-information." 32 Fragmented discourse, especially when it distracts or misguides the public from what they must do to protect themselves and others, has the potential to worsen the impact of a pandemic.

The purpose of this study was to demonstrate the use of SNA to understand public discourse on Twitter around the novel coronavirus (COVID-19). We focused on key public statements ("communication events") made by the WHO and its Director-General Dr. Tedros Ghebreyesus during the pandemic. As the leading international organization in the management of pandemics, we predicted that the accounts for the WHO (@WHO) and Dr. Ghebreyesus (@drtedros) would be leading the conversations about the coronavirus on Twitter. We expected the network to be highly centralized, with@WHO and @drtedros at the center of the conversation. Because networks are not static and public discourse on Twitter evolves, we expected the diameter and the modularity measures of the network to increase as more people tweeted about the issue and the discussion became more fragmented. Specifically, we had the following research questions: (1) What is the location of @WHO and @drtedros in the network of conversations around the coronavirus? Specifically, do they appear as central or core opinion leaders?; (2) Who are other opinion leaders on this topic on Twitter?; (3) How has the network changed across time, if at all?; (4) What inferences can we draw from the network properties regarding the successful transmission and adoption of public health messages on Twitter?

\section{METHODS}

For this study, we focused on conversations on Twitter around the novel coronavirus (COVID-19) from late January to early June of 2020. We used Netlytic (https://netlytic.org/), a Web-based software that collects publicly available data from social media sites, such as Twitter, and helps researchers build, visualize, and analyze communication networks using SNA. ${ }^{33,34}$ We focused on 3 key communication events: (1) the WHO's announcement of the novel coronavirus as a Public Health Emergency of International Concern (PHEIC) on January 30, (2) the WHO's declaration of COVID-19 as a pandemic on March 11, and (3) the WHO's updated assessment of the pandemic as a continued global threat on June 8, after surpassing 7 million cases and 400,000 deaths worldwide. For the first event, we used the search term "coronavirus" (the official naming of COVID-19 occurred on February 11). For the second and third events, we used the search terms "coronavirus" and "\#COVID19," a widely used hashtag, to collect tweets containing these terms. We set Netlytic to automatically collect tweets every 15 minutes on the dates specified. Every instance of data collection returned a maximum of 1000 tweets. The data collection generated 3 separate datasets of 100,000 tweets each, which is the maximum amount of records that Netlytic allows per dataset for data visualizations.

We examined the name network to determine the location of @WHO, @drtedros, and other actors in the network. Netlytic defines a name network as "a communication network built from mining personal names in the messages." ${ }^{35}$ Netlytic will extract each@name mentioned in a tweet and plot them in the network graph as separate nodes with edges or ties connecting the message author @ name to each of the @ names (s)he/they mentioned in the tweet. Twitter is a directed network, which means network visualizations of Twitter data will show a tie or an edge when a user mentions or replies to another user, not if they are following each other. That is different from Facebook, which is an undirected network and would show connections between "friends" as ties or edges.

For the social network visualizations, we used the Distributive Recursive Layout (DrL), which is "a force-directed graph layout, effective for visualizing large networks. ${ }^{35}$ In this layout, long edges are hidden to highlight clusters or communities of conversation. Clusters are groups of nodes that share a particular characteristic (eg, geographic location, sub-topic, or theme). These communities appear on the graph as round or oval shapes. The DrL layout is well-suited for visualizing all the different conversations happening over time and identifying who is at the center of each conversation. In some cases, the conversations are unrelated, with the only commonality 
being the search term or hashtag used. We examined diameter, density, reciprocity, centralization, and modularity to understand the topology of the network. We inspected who was mentioned the most, who posted the most, and who were retweeted the most to assess influence.

Finally, we also wanted to examine different network properties that might affect the successful dissemination and adoption of public health messages. Network properties include measures such as diameter, density, reciprocity, centralization, and modularity. ${ }^{35}$ The diameter measures the longest distance between 2 users in the network, counted in the number of nodes or unique Twitter user accounts (@name), that it takes to get from 1 participant to the other. Density measures how close nodes are in a network, while reciprocity measures 2-way communication or how much nodes are talking to each other. Centralization measures the extent to which a few nodes dominate the conversation. Each node has a centrality measure: indegree (based on times it has been mentioned or replied to), outdegree (based on times it has mentioned or replied to others), and total degree (the sum of both). Finally, modularity measures the fragmentation of a network into distinct communities. For all of these measures, values range from 0 (lowest) to 1 (highest). For example, modularity values closer to 1 "indicate clear divisions between communities," whereas values less than 0.5 suggest that the communities "overlap more; the network is more likely to consist of a core group of nodes." 35 A high modularity value reflects greater separation between communities of conversation. A network that is low in centrality means that there are many moderators and opinion leadership is less centralized, making it harder for public health officials and agencies to lead the conversation or have control of the message. Networks that are close-knit and homophilous, where people are similar to each other, tend to facilitate public health information dissemination and adoption of health behaviors. ${ }^{36,37}$ On the other hand, heterogeneous networks that are more spread out (larger in diameter), loosely connected (low in density and reciprocity), and fragmented (high in modularity) could lead to slower diffusion of information and threaten widespread adoption of scientifically sound health recommendations.

\section{RESULTS}

\section{Communication Event 1: WHO Declares the Novel Coronavirus a Public Health Emergency of International Concern}

One-hundred thousand tweets including "coronavirus" by 89,690 unique posters were collected from January 30, 2020, at 7:53 AM (AST) to January 31, 2020, at 8:54 AM (AST). The DrL visualization (Figure 1) shows the nodes with the highest total degree centrality in a network of 13,872 posters with 24,113 ties (including self-loops). A total of 97,920 unique @names were mentioned or replied to within the tweets. The top 5 nodes mentioned were @WHO $(N=1985)$,
$@$ drtedros $(N=348), @$ youtube $(N=268)$, @realdonaldtrump $(N=217)$, @cdcgov $(N=119)$, and $@_{c n n}(N=100)$. The network diameter is 7 nodes. The density measure $(N=0.00)$, the reciprocity measure $(N=0.00)$, and the centralization measure $(N=0.04)$ are all low. The modularity measure $(N=0.94)$ is high. Six of the top posters, defined as those users who generated the greatest number of tweets with the term "coronavirus" are accounts that self-identify as a bot or as automated in their profile bio. Approximately $81 \%$ of messages in the network were direct retweets (RTs). The users most retweeted were @helloalegria $(N=2023), @$ spectatorindex $(N=1093)$, @ solineura $(N=845)$, @zornitsaxx $(N=837)$, and @realdonaldtrump $(n=787)$

\section{Communication Event 2: WHO Declares COVID-19 a Pandemic}

One-hundred thousand tweets including "coronavirus" and "\#COVID-19" by 89,176 unique posters were collected from March 11, 2020, at 1:28 PM (AST) to March 12, 2020, at 1:54 Am (AST). The DrL visualization (Figure 2) shows the nodes with the highest total degree centrality in a network of 22,306 posters with 36,509 ties (including self-loops). A total of 97,209 unique @names were mentioned or replied to within the tweets. The top 5 nodes mentioned were @drtedros ( $N=7574)$, @realdonaldtrump $(N=1845)$, @WHO $(N=1652)$, @kris_lovaas $(N=693)$, @potus $(N=508)$, and @ thespinofftv $(N=419)$. The network diameter is 24 nodes. The density measure $(N=0.00)$, the reciprocity measure $(N=0.00)$, and the centralization measure $(N=0.09)$ are all low. The modularity measure $(N=0.90)$ is high. Four of the top posters are accounts that self-identify as news aggregators in their profile bio, 2 self-identify as bots, and 4 seem to be individual users who have a high retweet ratio or post very frequently. Approximately $86 \%$ of the records in the dataset represented RTs. The users most retweeted were $@$ @WHO $(N=8988)$, @conflits_fr $(N=2782)$, @drdenagrayson $(N=2,165)$, @noticiasonu $(N=1,629)$, and @feelthepress $(\mathrm{N}=795)$.

\section{Communication Event 3: WHO Warns Countries That COVID-19 Continues to Be a Global Threat, Urges Them to Continue to Fight It Actively and not to Let Their Guard Down}

One-hundred thousand tweets including "coronavirus" and “\#COVID19" by 90,014 unique posters were collected from June 8, 2020, at 7:58 PM (AST) to June 9, 2020, at 8:24 PM (AST). The DrL visualization (Figure 3) shows the nodes with the highest total degree centrality in a network of 25,295 posters with 52,031 ties (including self-loops). A total of 111,069 unique @names were mentioned or replied to within the tweets. The top 5 account names mentioned were @realdonaldtrump $(N=914)$, @secretarycarson $(N=636)$, @nicolasmaduro $(N=346)$, @lopezobrador $(N=342)$, and @WHO $(N=340)$. Tweets mentioning @secretarycarson are all retweets of @ $@$ realdonaldtrump thanking him. ${ }^{38}$ The 
FIGURE

Name Network (DrL) for the Term "Coronavirus" With the Top Names Mentioned in the Description Field of Tweets Collected from January 30, 2020, at 7:53 AM (AST) to January 31, 2020, at 8:54 AM (AST). We used Netlytic to generate this network visualization.

network diameter is 25 nodes. The density measure $(N=0.00)$, the reciprocity measure $(N=0.00)$, and the centralization measure $(N=0.01)$ are all low. The modularity measure $(N=0.96)$ is high. The top posters included a merchandise store, 3 news organizations or news aggregators, a journalist, a freelance writer, an entertainment podcast, and an individual account with a high retweet ratio. Approximately $78 \%$ of the records in the dataset represented RTs. The users most retweeted were @ $\mathrm{kyn} \_$joy $(N=1003)$, @youranoncentral $(N=761)$, @drjcofthedc $(N=716)$, @realdonaldtrump $(N=635)$, and @reaganschmagan $(N=438)$.

\section{DISCUSSION}

While @WHO and @drtedros had the highest total degree centrality in the first 2 events, the networks themselves were highly decentralized. For the first event, @WHO was the most mentioned name in the network within the description field of tweets, followed by @drtedros. For the second event, @drtedros was the most mentioned name, followed by @ realdonaldtrump and @WHO. This is not surprising given the importance of the WHO declaring the novel coronavirus a PHEIC on January 30 and a pandemic on March 11. The press conferences held by Dr. Ghebreyesus and the WHO were successful in attracting the attention of Twitter users following the coronavirus. What is interesting is the increasing attention earned by government figures as the pandemic continued to evolve. For the third event, @realdonaldtrump was the most mentioned name in the network, followed by other government figures from the United States, Venezuela, and Mexico. @WHO appears as the fifth most mentioned name in the network, and @drtedros was absent from the list of the top 30 mentioned names altogether. By early June, conversations around COVID-19 had become much more political than public health-focused, almost leaving @WHO and @drtedros outside of the conversation. 
Name Network (DrL) for the Term "Coronavirus" and "\#COVID19," Showing Only the 5 Main Clusters With the Top Names Mentioned in the Description Field of Tweets Collected From March 11, 2020, at 13:28 PM (AST) to March 12, 2020, at 1:54 AM (AST). We used Netlytic to generate this network visualization.

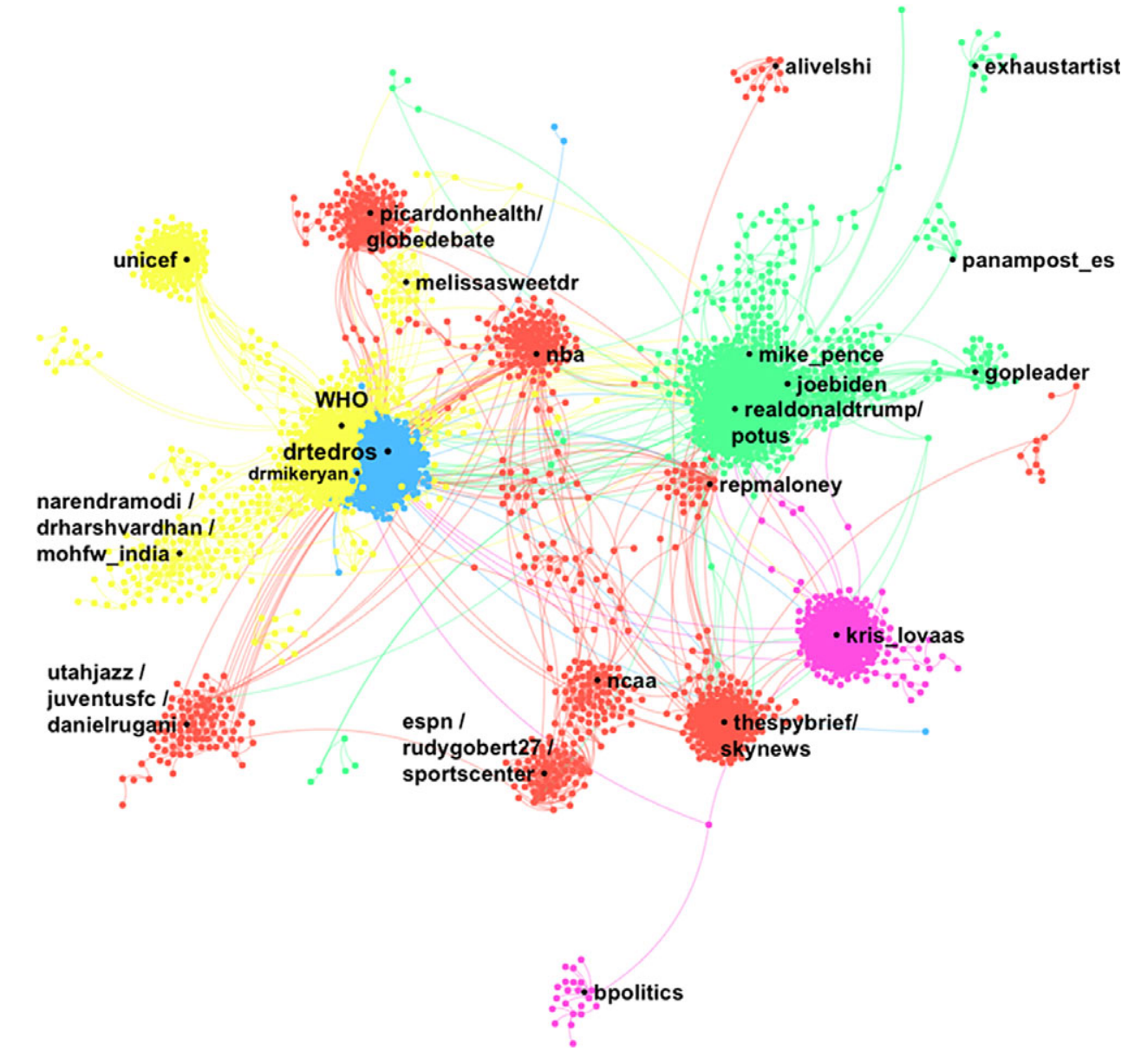

At the same time, the number of mentions must be interpreted in the context of the network. For example, the number of mentions of @WHO represents less than 2\% of all messages in the datasets for the first 2 events, and less than $0.5 \%$ of all messages for the third event. Mentions of @drtedros during the second event accounted for $7.5 \%$ of all messages in the network. The centralization values for all 3 network visualizations were very low $(N \leq 0.09)$, which means the networks were highly decentralized. At the same time, the number of messages mentioning the top @names in each network visualization does not account for a significant portion of the dataset; therefore, it is difficult to label any of the actors as core opinion leaders. Additionally, @names mentioned reflect who people are talking about the most, not who is doing most of the talking. Many of the mentions are negative in sentiment; they appeared to be more critical than supportive of actors' messages. Because of this, while @WHO and @drtedros received the most attention in the first 2 events, we conclude that the network of conversations about the coronavirus on Twitter is highly fractured and without clear leadership.

Applying the term conversation is also problematic since we found that 78 to $86 \%$ of tweets posted during each time frame were direct retweets of messages posted by others. This is consistent with the low reciprocity measures that we got; most people were not having conversations but rather broadcasting information and amplifying others' messages. Retweets are considered a form of 1-way communication and do not usually lead to conversations. At the same time, retweets allow us to identify whose messages are being amplified the most and can serve as a measure of indirect influence and opinion leadership. When we looked at retweets, some of the actors changed but their level of influence was still small compared with the size of the network. For example, while the most talked-about 


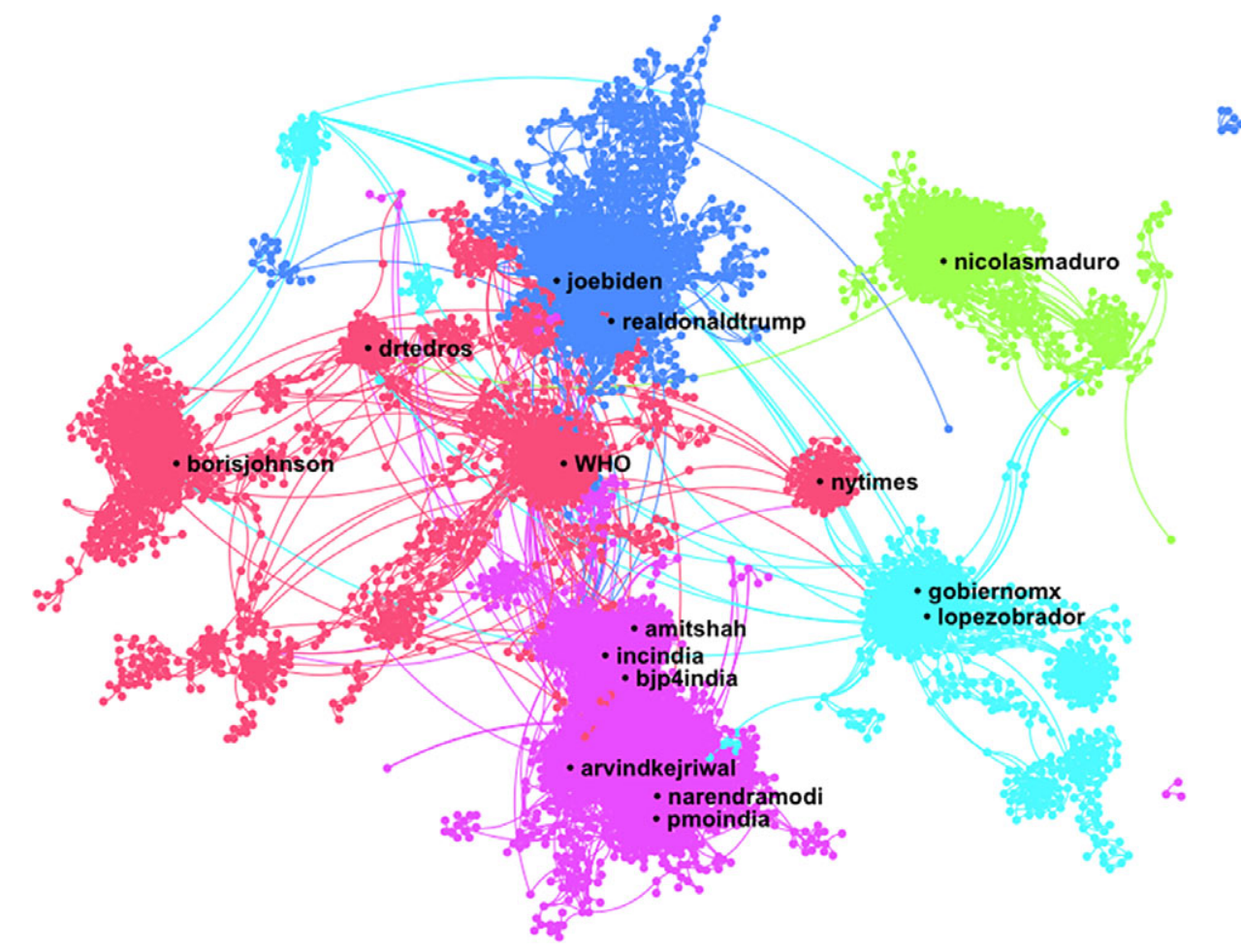

@names for the first event were @WHO and @drtedros, the most retweeted user was @ helloalegria, whose tweet calling out racist behavior toward Chinese people in the context of the coronavirus has been replied to 925 times, retweeted over 82,500 times and liked over 257,400 times. ${ }^{39}$ The tweet was in response to a video post from another user with the caption, "This is RACIST." 40 That tweet has been replied to over 2500 times, retweeted over 24,100 times, and liked over 89,100 times. The video itself has more than 5.1 million views. In comparison, while being the top name mentioned in the network for the first event, the most retweeted post from @WHO has been replied to 627 times, retweeted 894 times, and liked over 1300 times since January 30.41

The most retweeted post by @drtedros for the second event has been replied to 605 times, retweeted over 4200 times, and liked over 3800 times. ${ }^{42}$ The video included in the post, however, has over 2 million views. In summary, the individual's tweet commenting on the racialization of the virus got more traction than the official announcement made by Dr. Ghebreyesus and the WHO declaring COVID-19 a pandemic. Our analysis showed that in 2 of the 3 events, public health agencies, which should be leading the risk communication charge, were being second to individual social media influencers. This is concerning given the accompanying misinformation epidemic that has surrounded COVID-19 as evident by recent infodemiological studies in this area. ${ }^{29,43-47}$ The issue of misinformation is one that is worthy of its own study. The WHO defines an infodemic as "an overabundance of information-some accurate and some not-rendering it difficult to find trustworthy sources of information and reliable guidance," which in turn could have detrimental effects on the population and public health efforts to combat the virus itself. ${ }^{48}$ If public health agencies are to successfully combat the infodemic surrounding COVID-19, then it is critical that they lead the conversation.

We also observed changes in the network structure as the pandemic evolved, although not on all measures. The most significant change observed was in the network diameter, which went from 7 to 25 nodes from the first to the third events. This is not surprising with more people talking about COVID-19. More topics lead to fracturing into clusters or niches within the broader social network that forms around those topics. This, in turn, increases the diameter of the network, particularly when nodes are loosely connected, and people are not necessarily speaking to each other. Centralization 
was slightly higher for the second event, when WHO declared COVID-19 a pandemic, although the value was still very low. Modularity always remained high, increasing from 0.94 for the first event to 0.99 for the third event, representing increased fragmentation of the network. Density and reciprocity remained at 0.00 throughout all 3 events. There could be several explanations for this. First, the size of the datasets might be too large to get high values of density and reciprocity for the network as a whole, even though there might be individual clusters in the network that are higher in density and reciprocity. Another consideration is whether the topic lends itself to a conversation or is a news item that users are more likely to share with their followers, with commentary or as a direct retweet. Another possible explanation is that many more people may be using Twitter for broadcasting purposes rather than for being social and talking to others.

In addition to @WHO and @drtedros, we found that @realdonaldtrump, the verified personal account for US President Donald Trump, was the other node consistently and prominently mentioned throughout the pandemic, moving from the fourth most mentioned in the first event to the most mentioned in the third event. His tweets concerning the coronavirus were among the most retweeted in the first and third events. However, as discussed above, not all mentions were positive; many of the messages mentioning @realdonaldtrump were negative in sentiment. Of interest, @ cdcgov, the verified account of the Centers for Disease Control in the United States, appeared among the top 5 mentioned names on January 30, but not for the other 2 events. Beyond the most mentioned names, some themes emerged in the network visualizations. For the second event, localized conversations included a cluster focusing on US politics, another focused on the impact of COVID-19 on sports, specifically basketball, a media-moderated debate in Canada, and one focused on COVID-19 news in India. For the third event, there are conversations clustered by geographic location in India, Mexico, the United Kingdom, and Venezuela, most of them focusing on government actors. Other than its impact on the world of sports, the conversations around COVID-19 turned political in several countries, not just the United States. This is concerning given that the politicization of a virus threatens the credibility of scientifically based public health information and the effectiveness of public health officials and health agencies worldwide charged with doing risk communication.

Based on previous research studies looking at network topologies, the structure of these networks (highly decentralized, fragmented, and loosely connected) will hinder the successful dissemination of risk communication by public health officials and health agencies across the network. The high measure of modularity shows how fragmented the public's attention is on this topic. The growing diameter size, paired with low density, low reciprocity and high modularity, make it difficult for a topic to grab everyone's attention, either because participants do not care, and if they do, they might not feel motivated to comment on or share that content. What seems important from a risk communication standpoint is that looking at basic social media metrics might create a misleading picture of the effectiveness of risk communication efforts on social media if not analyzed within the context of the larger network. Basic social media metrics do not provide that information. SNA of conversations on social media should be an integral part of how public health officials and agencies plan, monitor, and evaluate risk communication efforts.

Our study has some limitations. First, due to Twitter's application programming interface restrictions, Netlytic limits data collection to 1000 tweets every 15 minutes. In other words, the tweets analyzed do not represent all of the tweets that were posted during the 3 communication events. Second, the low density and reciprocity measures that we saw across all communication events may not be unique to these networks; rather it could be representative of how people view and use Twitter, for news gathering and broadcasting, as opposed to other platforms like Facebook, which lend themselves more to conversations with others. Third, just because users are not engaging with content on Twitter does not mean that they are not commenting, sharing and/or adopting recommendations from public health officials and agencies through other platforms or offline.

\section{CONCLUSIONS}

In this study, we used SNA to examine and understand public discourse on Twitter around 3 key announcements made by the WHO during the COVID-19 pandemic. Specifically, we were interested in demonstrating the use of SNA to understand the network of conversations and actors regarding the novel coronavirus, identify potential roadblocks in the successful dissemination and adoption of health information, and realign risk communication messaging accordingly. We found that the network of conversations around COVID-19 is highly decentralized, fragmented, and loosely connected; these properties can hinder the successful dissemination of public health information. Also, competing conversations, misinformation, and other distractions by politically motivated actors can hamper risk communication efforts by public health officials and health agencies in a way that imperils public health. It is important, then, that public health agencies monitor communication on social media beyond basic quantitative social media metrics and text or content analyses. We recommend the integration of SNA as a best practice in risk communication on social media. This extended view recognizes that the space in which the discussion over COVID-19 takes place is one that is quite varied and includes diffuse users who are not only geographically diverse but also who represent divergent points of view and interest in this subject, ranging from scientific and public health experts to ordinary citizens who are seeking and sharing information to users who intentionally spread disinformation. Doing so will help public 
health agencies understand who is mediating the discussion regarding COVID-19 and what they can do to fight the accompanying infodemic.

\section{About the Authors}

Communication Department, Loyola University Maryland (Drs Pascual-Ferrá, Alperstein) and Department of Environmental Health $\mathcal{G}$ Engineering, Johns Hopkins Bloomberg School of Public Health (Dr Barnett)

Correspondence and reprint requests to Paola Pascual-Ferrá, 4501 N. Charles Street, Baltimore MD 21210 (e-mail: ppascualferra@loyola.edu).

\section{REFERENCES}

1. U.S. Department of Health and Human Services. Communicating in a crisis: risk communication guidelines for public officials. Washington, DC: US Department of Health and Human Services, 2002. https:/www.hsdl.org/ ?view\&did=440159. Accessed March 5, 2020.

2. Schiavo R. Health Communication: From Theory to Practice. 2nd ed. San Francisco, CA: Jossey-Bass; 2014.

3. World Health Organization. Risk communication saves lives \& livelihoods: pandemic influenza preparedness framework (brochure). https://www.who. int/risk-communication/PIP_brochure_EN_lo.pdf?ua=1. Accessed March 5, 2020.

4. World Health Organization. The Emergency Communications Network (ECN). https://www.who.int/risk-communication/emergency-response/. Accessed March 5, 2020.

5. Katz RL, Fischer JE. The revised International Health Regulations: a framework for global pandemic response. Glob Health Gov. 2010;3(2):1-18. http://www.ghgj.org/Katz\%20and\%20Fischer_The\%20Revised\%20Inter national\%20Health\%20Regulations.pdf. Accessed March 5, 2020.

6. Burson-Marsteller. Twitter is the prime social media network for world leaders. PR Newswire website. https://www.prnewswire.com/news-releases/ twitter-is-the-prime-social-media-network-for-world-leaders-300466300.html. Accessed March 11, 2020.

7. Ritterman J, Osborne M, Klein E. Using prediction markets and Twitter to predict a swine flu pandemic. In Proceedings of the 1st International Workshop of Mining Social Media. 2009;9:9-17 https://pdfs.semanticscholar.org/6e8f/ 50acc4023431a76e518f4f37ff02e89c7714.pdf. Accessed March 5, 2020.

8. Ahmed W, Bath PA, Sbaffi L, et al. Novel insights into views towards H1N1 during the 2009 Pandemic: a thematic analysis of Twitter data. Health Info Libr J. 2019;36(1):60-72. doi: 10.1111/hir.12247

9. McNeill AR, Briggs P. Understanding Twitter influence in the health domain: a social-psychological contribution. In Proceedings of the 23rd International Conference on World Wide Web. 2014:673-678. doi: $10.1145 / 2567948.2579280$

10. Signorini A, Segre AM, Polgreen PM. The use of Twitter to track levels of disease activity and public concern in the U.S. during the influenza A H1N1 pandemic. PLoS One. 2011;6(5):e19467. doi: 10.1371/journal. pone.0019467

11. Kostkova P, Szomszor M, St. Louis C. \#swineflu: The use of Twitter as an early warning and risk communication tool in the 2009 swine flu pandemic. ACM Trans Manag Inf Syst. 2014;5(2):1-25. doi: 10.1145/ 2597892

12. Szomszor M, Kostkova P, St Louis C. Twitter informatics: tracking and understanding public reaction during the 2009 swine flu pandemic. In 2011 IEEE/WIC/ACM International Conferences on Web Intelligence and Intelligent Agent Technology. 2011;1:320-323. doi: 10.1109/WI-IAT. 2011.311

13. McNeill A, Harris PR, Briggs P. Twitter influence on UK vaccination and antiviral uptake during the 2009 H1N1 pandemic. Front Public Health. 2016;4:26. doi: 10.3389/fpubh.2016.00026
14. Chew C, Eysenbach G. Pandemics in the age of Twitter: content analysis of tweets during the 2009 H1N1 outbreak. PLoS One. 2010;5(11):e14118. doi: 10.1371/journal.pone.0014118

15. Hughes A, Wojcik S. 10 facts about Americans and Twitter. Pew Research Center website. https://www.pewresearch.org/fact-tank/2019/08/02/10. facts-about-americans-and-twitter/. Accessed March 11, 2020.

16. Pfefferbaum B, North CS. Mental health and the Covid-19 pandemic. N Engl J Med. 2020;383(6):510-512. doi: 10.1056/NEJMp2008017

17. Nagler R, LoRusso S. Conflicting information and message competition in health and risk messaging. Oxford Research Encyclopedia of Communication. https://oxfordre.com/communication/view/10.1093/acrefore/978019 0228613.001.0001/acrefore-9780190228613-e-292. Published July 27, 2017. Accessed May 4, 2020.

18. Wu L, Ahn H. Making sense of conflicting health information: an exploratory study. In Proceedings of the American Society for Information Science and Technology. 2010;47(1):1-9. doi: 10.1002/meet.14504701178

19. Keeley D, Baxter N. Conflicting asthma guidelines cause confusion in primary care. BMJ. 2018;360:k29. doi: 10.1136/bmj.k29

20. Nagler RH, Yzer MC, Rothman AJ. Effects of media exposure to conflicting information about mammography: results from a populationbased survey experiment. Ann Behav Med. 2019;53(10):896-908. doi: 10.1093/abm/kay098

21. Nagler RH. Adverse outcomes associated with media exposure to contradictory nutrition messages. J Health Commun. 2014;19(1):24-40. doi: 10. 1080/10810730.2013.798384

22. Lupton DA. From complacency to panic: AIDS and heterosexuals in the Australian press, July 1986 to June 1988. Health Educ Res. 1992;7(1):9-20. doi: $10.1093 /$ her/7.1.9

23. Covello VT, Peters RG. Women's perceptions of the risks of age-related diseases, including breast cancer: reports from a 3-year research study. Health Commun. 2002;14(3):377-395. doi: 10.1207/S15327027HC1403_5

24. Cozzens MD, Contractor NS. The effect of conflicting information on media skepticism. Commun Res. 1987;14(4):437-451. doi: 10.1177/00936 5087014004004

25. Ranney LM, Jarman KL, Baker HM, et al. Factors influencing trust in agencies that disseminate tobacco prevention information. J Prim Prev. 2018;39(2):99-116. doi: 10.1007/s10935-018-0501-3

26. Isa D, Himelboim I. A social networks approach to online social movement: Social mediators and mediated content in \#FreeAJStaff Twitter network. Soc Media Soc. 2018;4(1):1-14. doi: 10.1177/2056305118760807

27. LaPoe V, Jain P, LaPoe B, et al. Politics and power of a pandemic: analysis of COVID-19 tweets during the early stages of public awareness. Social Sciences \& Humanities Open (SSRN website). https://ssrn.com/ abstract=3603751. Accessed August 23, 2020.

28. Rufai SR, Bunce C. World leaders' usage of Twitter in response to the COVID-19 pandemic: a content analysis. J Public Health (Oxf). 2020; 42(3):510-516. doi: 10.1093/pubmed/fdaa049

29. Park HW, Park S, Chong M. Conversations and medical news frames on Twitter: infodemiological study on COVID-19 in South Korea. J Med Internet Res. 2020;22(5):e18897. doi: 10.2196/18897

30. Wasserman S, Faust KL. Social Network Analysis: Methods and Applications. New York: Cambridge University Press; 1994.

31. Carrington PJ, Scott J, Wasserman S. Models and Methods in Social Network Analysis. New York: Cambridge University Press; 2005.

32. Nahon-Serfaty I. The disruptive consequences of discourse fragmentation in the organization and delivery of health care: a look into diabetes. Health Commun. 2012;27(5):506-516. doi: 10.1080/10410236. 2011.618426

33. Gruzd A. Netlytic: software for automated text and social network analysis. http://Netlytic.org. Accessed July 5, 2020.

34. Netlytic.org. About. https://netlytic.org/home/?page_id=10834. Accessed July 1, 2020.

35. Netlytic.org. Network analysis/visualization. https://netlytic.org/home/? page_id=2\#cmtoc_anchor_id_5. Accessed July 1, 2020.

36. Centola D. The spread of behavior in an online social network experiment. Science. 2010;329(5996):1194-1197. doi: 10.1126/science.1185231 
37. Centola D. An experimental study of homophily in the adoption of health behavior. Science. 2011;334(6060):1269-1272. doi: 10.1126/science. 1207055

38. @ realdonaldtrump. I am allocating \$2.96 BILLION in Emergency Solutions Grants to support homeless Americans and those at risk of becoming homeless because of job or wage loss, or illness due to COVID-19. We are taking care of our Nation's most vulnerable citizens. Thanks @SecretaryCarson! https://twitter.com/realDonaldTrump/status/ 1270411233733287936. Accessed July 6, 2020.

39. @helloalegria. coronavirus has been racialised as a "chinese" illness and for this reason chinese people, regardless of their proximity to wuhan are being treated like carriers of the virus. similar to how ebola was subtly touted as an "african" illness [included embedded tweet by @Blayofficial]. https:// twitter.com/helloalegria/status/1222650786393608192?lang=en. Accessed July 6, 2020.

40. @Blayofficial. This is RACIST [included embedded video]. https://twitter. com/Blayofficial/status/1222648026663178240. Accessed July 6, 2020.

41. @WHO. The International Health Regulations Emergency Committee on the new \#coronavirus (2019-nCoV) has reconvened today. Read more about the Emergency Committee http://bit.ly/2RjJugm [included photo showing Director-General Dr. Tedros Ghebreyesus] https://twitter.com/ who/status/1222865860483829760?lang=en. Accessed July 6, 2020.

42.@DrTedros..@WHO is deeply concerned by the alarming levels of the \#coronavirus spread, severity \& inaction, \& expects to see the number of cases, deaths \& affected countries climb even higher. Therefore, we made the assessment that \#COVID19 can be characterized as a pandemic [included video of Director-General Dr. Tedros Ghebreyesus and embedded tweet from @WHO]. https://twitter.com/drtedros/status/ 1237800182235922434 ?lang=en. Accessed July 6, 2020.

43. Cuan-Baltazar JY, Muñoz-Perez MJ, Robledo-Vega C, et al. Misinformation of COVID-19 on the Internet: infodemiology study. JMIR Public Health Surveill. 2020;6(2):e18444. doi: 10.2196/18444

44. Hernández-García I, Giménez-Júlvez T. Assessment of health information about COVID-19 prevention on the Internet: infodemiological study. JMIR Public Health Surveill. 2020;6(2):e18717. doi: 10.2196/18717

45. Rovetta A, Bhagavathula AS. COVID-19-related web search behaviors and infodemic attitudes in Italy: infodemiological study. JMIR Public Health Surveill. 2020;6(2):e19374. doi: 10.2196/19374

46. Moon H, Lee GH. Evaluation of Korean-Language COVID-19-related medical information on YouTube: cross-sectional infodemiology study. J Med Internet Res. 2020;22(8):e20775. doi: 10.2196/20775

47. Hou Z, Du F, Zhou X, et al. Cross-country comparison of public awareness, rumors, and behavioral responses to the COVID-19 epidemic: infodemiology study. J Med Internet Res. 2020;22(8):e21143. doi: 10.2196/ 21143

48. United Nations Department of Global Communications. UN tackles 'infodemic' of misinformation and cybercrime in COVID-19 crisis. United Nations website. Accessed 'https://www.un.org/en/uncoronavirus-communications-team/un-tackling-'infodemic'-misinformationand-cybercrime-covid-19. August 23, 2020. 